\title{
Multicanonical evaluation of the tails of the probability density function of semiconductor optical amplifier output power fluctuations
}

Tromborg, Bjarne; Reimer, Michael; Yevick, David

Published in:

I E E E Journal of Quantum Electronics

Link to article, DOI:

10.1109/JQE.2009.2026724

Publication date:

2010

Document Version

Publisher's PDF, also known as Version of record

Link back to DTU Orbit

Citation (APA):

Tromborg, B., Reimer, M., \& Yevick, D. (2010). Multicanonical evaluation of the tails of the probability density function of semiconductor optical amplifier output power fluctuations. I E E E Journal of Quantum Electronics, 46(1), 57-61. https://doi.org/10.1109/JQE.2009.2026724

\section{General rights}

Copyright and moral rights for the publications made accessible in the public portal are retained by the authors and/or other copyright owners and it is a condition of accessing publications that users recognise and abide by the legal requirements associated with these rights.

- Users may download and print one copy of any publication from the public portal for the purpose of private study or research.

- You may not further distribute the material or use it for any profit-making activity or commercial gain

- You may freely distribute the URL identifying the publication in the public portal 


\title{
Multicanonical Evaluation of the Tails of the Probability Density Function of Semiconductor Optical Amplifier Output Power Fluctuations
}

\author{
Bjarne Tromborg, Michael Reimer, Student Member, IEEE, and David Yevick, Fellow, IEEE
}

\begin{abstract}
This paper presents a multicanonical Monte Carlo method for simulating the tails of a probability density function (pdf) of the filtered output power from a semiconductor optical amplifier down to values of the order of $10^{-40}$. The influence of memory effects on the pdf is examined in order to demonstrate the manner in which the calculated pdf approaches the true pdf with increasing integration time. The simulated pdf is shown to be in good agreement with a second-order analytic expression for the pdf.
\end{abstract}

Index Terms-Microcanonical Monte Carlo, noise, optical communications, semiconductor optical amplifiers (SOAs).

\section{INTRODUCTION}

$\mathbf{T}$ HE multicanonical Monte Carlo (MMC) method is an iterative method for numerical calculation of the probability density function (pdf) of a stochastic variable by biased simulations starting from a probe pdf distribution. Each iteration results in a new distribution that is used as input in the next iteration. Under certain conditions, which are usually not known precisely in advance, the iterated distributions converge toward the true distribution. The method was introduced by Berg and Neuhaus in 1992 [1] to analyze problems in statistical physics but has since been applied to many other fields. An excellent review is given by Berg in [2]. The main advantage of the MMC method is that it allows calculation of extremely low pdf values as for example down to $10^{-80}$ [3]. Such low values cannot be reached by unbiased Monte Carlo simulations within practical time limits, and it may even be difficult to derive the pdf from an analytic expression by double precision computation.

In the field of optical communications, the MMC method was first applied to calculate polarization-mode dispersion emulator statistics [4], and the example has since been widely used as a laboratory for studying different schemes of biasing Monte Carlo calculations [5]-[9].

Manuscript received February 11, 2009; revised June 03, 2009. Current version published December 04, 2009. This work was supported in part by the Natural Sciences and Engineering Research Council of Canada (NSERC), in part by the Centre for Electrophotonic Materials and Devices (CEMD), and in part by the Ontario Research and Development Challenge Fund (ORDCF).

B. Tromborg is with the Department of Photonics Engineering, DTU Fotonik, Technical University of Denmark, Lyngby 2800, Denmark (e-mail: btromborg@mail.dk).

M. Reimer and D. Yevick are with the Department of Physics and Astronomy, University of Waterloo, Waterloo, Ontario N2L 3G1, Canada (e-mail: yevick@uwaterloo.ca).

Digital Object Identifier 10.1109/JQE.2009.2026724
The MMC algorithm can also be employed to study the reduction in performance of optical communication systems arising from spontaneous emission noise generated in transmitters and optical amplifiers. The bit error rate (BER) of received signals, which is a figure of merit for system performance, can be obtained from the tails of the pdf distributions of the received optical power. The MMC method has been employed in a number of papers to calculate such distributions (see e.g., [10]-[15]). In [10], Holzlöhner and Menyuk applied the MMC method to simulate the pdf distributions of received marks and spaces of a $10 \mathrm{Gbit} / \mathrm{s}$ signal after transmission through a simulated underwater communication system with many fiber amplifiers. The signal noise resulting from spontaneous emission was added at each amplifier and coupled to the signal by the fiber nonlinearities in the succeeding transmission. A similar approach was implemented in [11] to study the noise properties of a dense WDM system. Bilenca and Eisenstein [12], [13] examined the pdf distribution of the marks of a digital signal after transmission through a nonlinear semiconductor optical amplifier (SOA) by MMC simulations, analytic methods, and experiments. In recent papers, Lakoba and Vasilyev [14], [15] applied the MMC methods to study the BER of the output signal from a $2 \mathrm{R}$ regenerator. Similar systems have been further analyzed in [16]. All these noise analyses propagate a signal from the input to the output by solving stochastic differential equations. They further consider systems with memory effects, either because of the presence of optical and/or electrical filters or because of the finite lifetime of the nonlinear processes.

In this article, we study the pdf of the filtered output power from a moderately saturated SOA for continuous-wave (CW) input power. This is a highly nonlinear system that additionally exhibits memory effects as a result of the presence of the filter as well as the finite carrier lifetime. We will incorporate such memory effects into the multicanonical formalism. Our analysis shows that it is sufficient to use an integration period which is approximately twice the sum of the carrier lifetime and the filter delay time. We also develop a procedure that yields accurate predictions for very low pdf values $\left(<10^{-16}\right)$ that may be of interest in future applications. Our method yields the pdf reliably even for values as low as $10^{-40}$. Finally, the MMC results for the pdf are shown to agree with calculations from analytic expressions in [17] when all second-order noise contributions are taken into account.

The paper is organized as follows. In Section II, we present the system of equations describing the SOA and the method for solving them. The MMC algorithm is introduced in Section III. 
In Section IV, the algorithm is applied to calculate the pdf of the SOA output power focusing on system memory effects and comparing two different starting conditions. Section V contains our conclusions.

\section{SOA OUTPUT POWER Simulations}

We model an SOA with a length of $L=500 \mu \mathrm{m}$ and perfectly AR-coated facets. The complex electric field $E(z, t)$, with longitudinal coordinate $z$ in the SOA waveguide, is normalized such that $|E(z, t)|^{2}$ is the power. The equations governing the time dependence of the electric field amplitude and gain are assumed to be of the standard form [17]-[19]

$$
\begin{aligned}
\frac{\partial E}{\partial z} & =\frac{1}{2}\left[g(1-i \alpha)-\alpha_{\mathrm{int}}\right] E+f_{E} \\
\frac{\partial g}{\partial t} & =\frac{g_{0}-g}{\tau_{s}}-\frac{g|E|^{2}}{\tau_{s} P_{\mathrm{sat}}} .
\end{aligned}
$$

Here $g$ is the modal gain, $\tau_{s}$ is the spontaneous carrier lifetime, $\alpha$ is the linewidth enhancement factor, and $\alpha_{\text {int }}$ is the waveguide loss. The function $f_{E}$ is a Langevin noise term, which describes the spontaneous emission noise. The time variable $t$ is a shifted time coordinate, $t=t_{\text {real }}-z / v_{\mathrm{g}}$, where $t_{\text {real }}$ is the real-time coordinate and $v_{\mathrm{g}}$ is the group velocity. $P_{\text {sat }}$ is the saturation power

$$
P_{\text {sat }}=\frac{A \hbar \omega_{0}}{a \tau_{s}}
$$

where $\hbar \omega_{0}$ is the photon energy, $A$ is the effective cross section area of the active region, and $a$ is the differential modal gain. We model the gain by a linear function of the carrier density $N$, i.e., $g(N)=a\left(N-N_{0}\right)$ with $N_{0}$ the carrier density at transparency. The unsaturated gain $g_{0}$, i.e., the steady-state gain when $|E|^{2}=0$, is

$$
g_{0}=a\left(\frac{I}{q V}+\frac{N_{0}}{\tau_{s}}\right)
$$

in which $I$ is the current injected into the SOA, $q$ is the elementary charge, and $V$ is the active volume.

The system of rate equations (1) and (2) are solved by a method which is discussed in detail in [20] and is based on the method of [21]. In order to implement the MMC method, we briefly present the main features of the algorithm for solving the equations. The SOA is divided into $N_{z}$ sections and an integrated gain

$$
h_{k}(t)=\int_{z_{k-1}}^{z_{k}} g\left(z^{\prime}, t\right) d z^{\prime}, k=1, \ldots, N_{z}
$$

is defined within each section with $z_{k}=k \Delta z$ and $\Delta z=L / N_{z}$. Neglecting the internal loss in the $k$ th section, $h_{k}(t)$ obeys the rate equation

$$
\frac{\partial h_{k}}{\partial t}=\frac{h_{0}-h_{k}(t)}{\tau_{s}}-\frac{\left|E_{k}(t)\right|^{2}}{\tau_{s} P_{\mathrm{sat}}}\left(e^{h_{k}(t)}-1\right)
$$

where $h_{0}=g_{0} \Delta z$, and $\left|E_{k}(t)\right|^{2}$ is the power input to the $k$ th section. The field input $E_{k+1}(t)$ is

$E_{k+1}(t)=E_{k}(t) \exp \left\{\frac{\left[h_{k}(t)(1-i \alpha)-\alpha_{\text {int }} \Delta z\right]}{2}\right\}+\sigma \sqrt{P_{\text {sat }}} n_{k}$.

The field at the input facet, $E_{1}(t)$, is set to a constant value. The loss that we neglected in (6) is included in (7), which is a good approximation for small $\Delta z$. The two equations are evolved over time intervals $\Delta t$ while a noise term $\sigma \sqrt{P_{\text {sat }}} n_{k}$ is added in each section to simulate spontaneous emission effects. The factors $n_{k}$ are independent Gaussian complex random variables with unit width and $\sigma$ is given by

$$
\sigma^{2}=\frac{\hbar \omega g n_{\mathrm{sp}} \Delta z}{2 P_{\mathrm{sat}} \Delta t} .
$$

Here $n_{\mathrm{sp}}$ is the population inversion factor. As in [17], we approximate $g n_{\mathrm{sp}}$ by $g_{s}+a N_{0}$, where $g_{s}(z)$ is the steady-state gain. The sampling of noise contributions with sampling time $\Delta t$ models a (sinc $)^{2}$-shaped spontaneous emission spectrum with bandwidth $B=1 /(2 \Delta t)$.

The field at the output facet, $E_{\text {out }}(t)$, is given by (7) for $k=$ $N_{z}$. It may be filtered to model an optical filter [17], but in the present article, we only include electronic filtering of the output power $P_{\text {out }}=\left|E_{\text {out }}\right|^{2}$ to model the receiver. The filtered output is

$$
P_{F}(t)=\int_{-\infty}^{t} F_{e}\left(t-t^{\prime}\right) P_{\text {out }}\left(t^{\prime}\right) d t^{\prime}
$$

where $F_{e}(t)$ is the filter response function. In our subsequent example, we average the output power over nine time samples corresponding to a simple low-pass filter.

\section{Multicanonical Procedure}

Our multicanonical analysis of the pdf of the filtered output power $P_{F}$ follows the technique of [10]. In particular, we first subdivide the relevant range of output powers, $\left[P_{\min }, P_{\max }\right]$, into $N_{b}$ histogram bins. A histogram representing the unnormalized pdf of $P_{F}$ is obtained by repeatedly solving (6) and (7) and assigning each result for $P_{F}$ to the appropriate histogram bin. In order to incorporate correctly memory effects, the integration time $T$, before $P_{F}$ is sampled, must exceed the sum of the filter response time and the largest lifetime of all the SOA processes that influence the power. The power thus depends on the set of Gaussian noise terms $\left\{n_{k}(t-j \Delta t) \mid k=1, \ldots, N_{z} ; j=\right.$ $\left.0, \ldots, N_{t}-1\right\}$, where $N_{t}=T / \Delta t$ is the number of time samples. We then form a real vector $\mathbf{x}$ in a state space of dimension $d=2 N_{z} N_{t}$ from the real and imaginary parts of the noise terms. The probability in state space is $\rho(\mathbf{x})=\prod_{\ell=1}^{d} \rho_{\ell}$, where $\rho_{\ell} \propto \exp \left(-x_{\ell}^{2} / 2\right)$ is the Gaussian distribution of the $\ell$ th component of $\mathbf{x}$.

In a standard unbiased Monte Carlo simulation, the vectors $\mathbf{x}$ in state space are sampled at random values in accordance with the Gaussian statistics of the components, but this approach makes it very time consuming to reach values of low pdf. The present MMC method is based on a Metropolis-Hastings algo- 
rithm [22], [23] that instead uses a biased sampling of a Markov chain of transitions. Each transition from $\mathbf{x}_{a}$ to $\mathbf{x}_{b}$ in state space is generated in such a manner that the transition probability $Q\left(\mathbf{x}_{b} \mid \mathbf{x}_{a}\right)$ satisfies the relation

$$
Q\left(\mathbf{x}_{b} \mid \mathbf{x}_{a}\right) \rho\left(\mathbf{x}_{a}\right)=Q\left(\mathbf{x}_{a} \mid \mathbf{x}_{b}\right) \rho\left(\mathbf{x}_{b}\right) .
$$

In particular, given an initial state $\mathbf{x}_{a}$, the succeeding state in the Markov chain is selected as follows. We introduce a vector

$$
\mathbf{x}=\mathbf{x}_{a}+\epsilon \Delta \mathbf{x}
$$

where $\epsilon$ is a fixed number of order one. The components $\Delta x_{\ell}$ of $\Delta \mathrm{x}$ are random numbers uniformly distributed in the interval $[-1 / 2,1 / 2]$. To generate the subsequent state $\mathbf{x}_{b}$, each component $x_{\ell}$ of $\mathbf{x}$ is accepted with probability $\min \left[1, \rho_{\ell}\left(x_{\ell}\right) / \rho_{\ell}\left(x_{a, \ell}\right)\right]$; otherwise, we set $x_{b, \ell}=x_{a, \ell}$. In this manner, the transition probability $Q\left(\mathbf{x}_{a} \mid \mathbf{x}_{b}\right)$ satisfies the relation (10).

The MMC applies an iterative technique in which the input to the $j$ th iteration is an estimate $P_{k}^{j}$ of the probability for the power to be in the $k$ th bin. A transition from $\mathbf{x}_{a}$ to $\mathbf{x}_{b}$ is accepted with probability $\min \left[1, P_{k_{a}}^{j} / P_{k_{b}}^{j}\right]$, where $k_{a}$ and $k_{b}$ are the bins that contain the powers $P_{F}\left(\mathbf{x}_{a}\right)$ and $P_{F}\left(\mathbf{x}_{b}\right)$. In the cases that the transition is accepted or rejected, the histogram is incremented by unity in bins $k=k_{b}$ or $k=k_{a}$, respectively, after which the succeeding transition occurs from $\mathbf{x}_{b}$ or $\mathbf{x}_{a}$.

In this manner, as the number of Markov steps and iterations increases, the histogram $H_{k}^{j}$ approaches a distribution proportional to $P_{k} / P_{k}^{j}$, where $P_{k}$ is the true pdf distribution. The estimate $P_{k}^{j+1}$ for the next iteration may therefore simply be given by $P_{k}^{j+1}=c_{j} H_{k}^{j} P_{k}^{j}$, where $c_{j}$ is a normalization constant. However, the accuracy can be further improved by calculating $P_{k}^{j+1}$ by recursion relations in terms of histograms and probability estimates from the $j$ th and former iterations [2], [5], [8], [10], [24]. We use the recursion relations given by (6a-b) in [10], which is a compact formulation of the algorithm of [24]. This and other optimization algorithms for the estimates are compared in [8]. While the initial distribution $P^{1}$ is generally chosen to be a constant function, in the calculation below increased accuracy and calculation speed can be achieved by instead employing an approximation to the pdf.

\section{NUMERICAL RESULTS}

In this section, the MMC algorithm is applied to simulate the pdf of the filtered output power from an SOA with a CW input power that moderately saturates the amplifier. The parameters of the example are presented in Table I. The length of the SOA is $500 \mu \mathrm{m}$ for which (6) and (7) accurately predict the behavior of the amplifier for $\Delta z=10 \mu \mathrm{m}$ corresponding to $N_{z}=50$. For a 100 ps carrier lifetime and 72 ps filter integration time, we expect that the total integration time $T$ should be chosen larger than $172 \mathrm{ps}$, i.e., $N_{t}=T / \Delta t>21$, to ensure that the pdf does not depend on $T$. The corresponding dimension of the state space must therefore be larger than 2100 . This agrees with calculations of the pdf for increasing integration time. Fig. 1

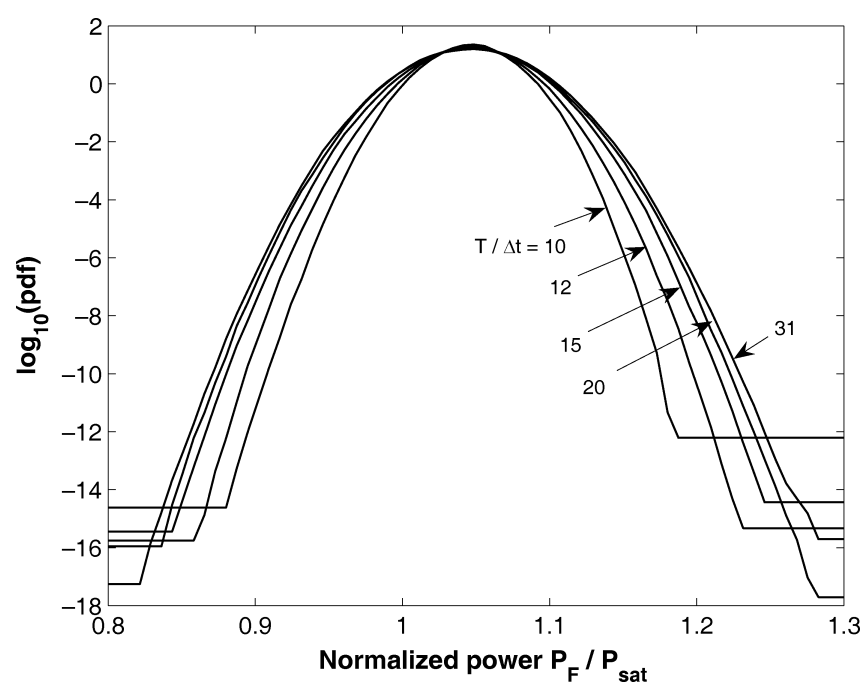

Fig. 1. pdf as a function of the normalized power $P_{F} / P_{\text {sat }}$ for integration times $T / \Delta t=10,12,15,20$, and 31 . The calculations employ eight 25,000 sample MMC iterations.

TABLE I

LIST OF PARAMETER VALUES

\begin{tabular}{lc}
\hline Parameter & Value \\
\hline Device length, $L[\mu \mathrm{m}]$ & 500 \\
Number of sections, $N_{z}$ & 50 \\
Carrier lifetime, $\tau_{s}[\mathrm{ps}]$ & 100 \\
Sampling time, $\Delta t[\mathrm{ps}]$ & 8 \\
Filter integration time, $9 \Delta t[\mathrm{ps}]$ & 72 \\
Number of bins, $N_{b}$ & 100 \\
Input power, [mW] & 0.009 \\
Saturation power, $P_{\text {sat }}[\mathrm{mW}]$ & 6 \\
Linewidth enhancement factor, $\alpha$ & 5 \\
Internal loss, $\alpha_{i n}\left[(\mu m)^{-1}\right]$ & $5 \cdot 10^{4}$ \\
Small signal gain, $g_{0}\left[(\mu m)^{-1}\right]$ & 0.0158 \\
Differential modal gain, $a\left[\mathrm{~m}^{2}\right]$ & $3.77 \cdot 10^{-20}$ \\
Carrier density at transparency, $N_{0}\left[\mathrm{~m}^{-3}\right]$ & $10^{24}$ \\
Operating wavelength, $[\mu \mathrm{m}]$ & 1.55 \\
\hline
\end{tabular}

demonstrates the manner in which the calculated pdf distributions for $N_{t}=10,12,15,20$, and 31 converge toward a final distribution as $N_{t}$ is increased. The distribution for $N_{t}=40$ (not shown) in fact is indistinguishable from the $N_{t}=31$ result within the resolution of the diagram. These MMC calculations employ eight 25,000 sample iterations together with a constant initial estimate $P^{1}$. The factor $\epsilon$ in (11) is fixed for each iteration and is chosen as $1.06^{j-1}$ for the $j$ th iteration. The photons in the SOA cavity reduce the effective carrier lifetime below $\tau_{s}$, which presumably explains the rapid convergence of the results in Fig. 1. In a standard unbiased Monte Carlo simulation, we do not have to worry about memory effects but can let the simulation run continuously and sample the power every $\Delta t$. This means that the MMC method loses a factor of $N_{t}$ in the total number of samples within a given time compared to the unbiased Monte Carlo method. Nevertheless, the latter method would only produce a pdf curve down to around $10^{-5}$ in the time used for calculating one of the curves in Fig. 1. 


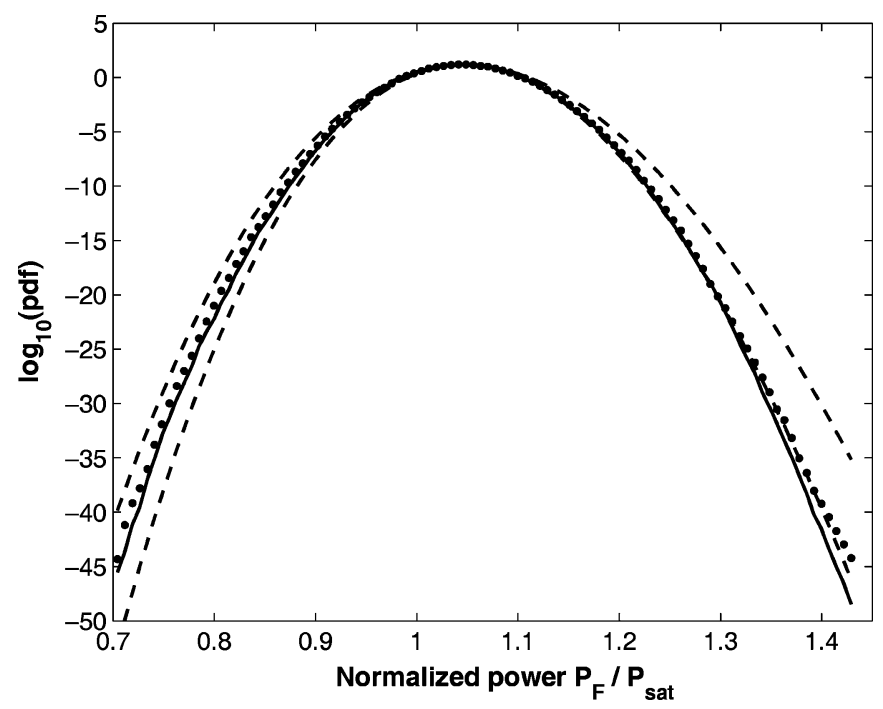

Fig. 2. Variation of the pdf with the normalized power $P_{F} / P_{\text {sat }}$. Analytic $\chi^{2}$-distributions with $1 / \lambda=1.7 \times 10^{-4}$ and $1.3 \times 10^{-4}$ (dashed curves) are shown together with the results of MMC calculations starting from the upper and lower of these curves (dotted and solid curves, respectively).

Based on the analysis in [17], we expect the pdf to be well approximated by the noncentral $\chi^{2}$-distribution

$$
\operatorname{pdf}\left(P_{F}\right)=\frac{\lambda P_{\text {sat }}}{2 P_{s}} e^{-(x+\lambda) / 2}\left(\frac{\lambda}{x}\right)^{1 / 4} I_{-1 / 2}(\sqrt{\lambda x})
$$

where $I_{\nu}$ is a modified Bessel function of the first kind, and $x$ is given in terms of the power $P_{F}$ by

$$
x=\lambda\left[\frac{P_{F}}{P_{s}}-\left(\frac{1}{\lambda_{0}}-\frac{1}{\lambda}\right)\right] .
$$

Here $P_{s}$ is the output power in the absence of noise $(\sigma=0)$, and $1 / \lambda\left(1 / \lambda_{0}\right)$ is the right side of (55) in [17] for $F(\omega)=F_{e}(\omega)$ $(F(\omega)=1)$. The filter transfer function $F_{e}(\omega)$ is in our case equal to $\left.\sum_{k=0}^{8} \exp (i k \omega \Delta t)\right) / 9$. We find $1 / \lambda_{0}=1.966 \times 10^{-3}$ and $1 / \lambda=1.457 \times 10^{-4}$ when the parameters of Table I are used. The distribution (12) is normalized such that the integral over the normalized power $P_{F} / P_{\text {sat }}$ is unity.

We have performed two MMC calculations starting from the noncentral $\chi^{2}$-distributions (12) for $1 / \lambda=1.7 \times 10^{-4}$ and $1 / \lambda=1.3 \times 10^{-4}$, which lie on either side of the distribution for $1 / \lambda=1.457 \times 10^{-4}$. The distributions are the dashed curves in Fig. 2, while the dotted and solid curves are the MMC results obtained from the upper and lower $\chi^{2}$ initial distributions, respectively. The MMC calculations employed eighteen 25,000 sample iterations with $T=31 \Delta t$. For the first eight iterations, the $\epsilon$-factor was set to $1.06^{j-1}$ while for the remainder we employed $\epsilon=1.3$. Evidently, we can reproduce the tails of the probability distribution down to $10^{-45}$. While on the low power side, the two curves approach each other from opposite directions as the number of iterations is increased, indicating that the actual distribution is situated between the two results; on the high power side, both curves are displaced to lower values so that the final result is expected to be less than or close to that indicated by the solid curve. We have therefore performed a third

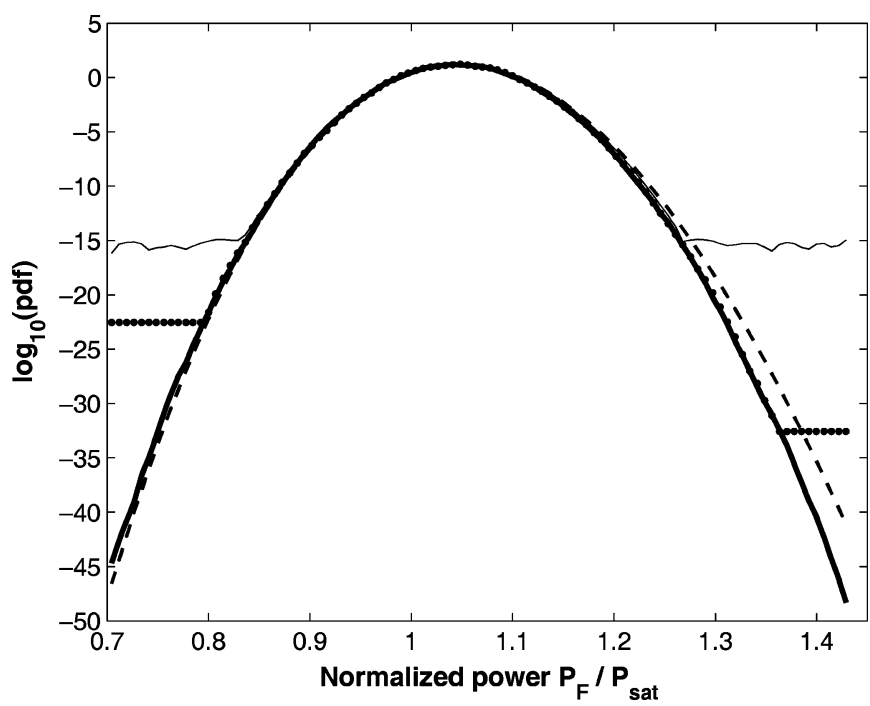

Fig. 3. Variation of the pdf with the normalized power $P_{F} / P_{\text {sat }}$. Results of an MMC calculation starting from the solid curve of Fig. 2 employing eight 25,000 sample iterations (thick solid curve) and an MMC calculation starting from a constant distribution and with eighteen 25,000 sample iterations (dotted curve). Analytic second-order small signal approximation to the pdf (thin solid curve) and the prediction of (12) with $1 / \lambda=1.457 \times 10^{-4}$ (dashed curve).

MMC calculation starting from the solid curve and employing eight 25,000 sample iterations. The result is shown as the thick solid curve in Fig. 3. On the low power side, it is close to the dotted curve of Fig. 2 and on the high power side, it is close to the solid curve of Fig. 2. For comparison, we have made an MMC calculation with eighteen 25,000 sample iterations starting from a constant distribution. It is shown as the dotted curve in Fig. 3 and agrees with the thick solid curve within $4 \mathrm{~dB}$ except for the ranges at low and high power where it is constant. In a pdf range from $10^{-4}$ on the low power side to $10^{-8}$ on the high power side, the agreement is within $1 \mathrm{~dB}$. Each 25,000 sample iteration requires approximately $7 \mathrm{~h}$ of CPU time on an AMD Athlon 64 $3200+$ computer, with the MATLAB "ode45" differential equation solver. While the computation time can be reduced in our calculations by instead applying Heun's method [12], the relative efficiency of the MATLAB routine increases if higher levels of numerical accuracy are required.

We also include in Fig. 3 a calculation employing the secondorder small signal expression for the pdf of [17]. This calculation is limited to an accuracy of $10^{-15}$ as a result of double precision rounding errors. However, above that level, it nearly coincides with the MMC curves on the low power side while it is only in moderate agreement on the high power side. The deviation is probably the result of the approximations contained in our calculation of second-order noise contributions. Finally, the dashed curve indicates the noncentral $\chi^{2}$-distribution for the first-order result with $1 / \lambda=1.457 \times 10^{-4}$. As in [17], we notice that the skewness of the $\chi^{2}$-distribution exceeds that of the simulated distribution.

\section{CONCLUSION}

We have examined the influence of memory effects on an MMC calculation of the pdf of detected output power from an 
SOA with CW input. Our calculations confirm that the integration time should be greater than all the time scales of the system.

By initializing our MMC calculations with approximate $\chi^{2}$-distributions, we have obtained a pdf distribution that was demonstrated to be accurate to probability densities of approximately $10^{-40}$ through a comparison of the resulting curves. The pdf distribution is also compared with a curve generated by instead employing a constant as the initial distribution. Within their common range, the two calculations agree within $4 \mathrm{~dB}$.

The MMC simulations are finally compared with the distribution obtained from the analytical expression for the pdf in [17], which is valid to second order in the spontaneous emission noise factor $\sigma^{2}$. The agreement is excellent on the low power side, but there is only moderate agreement on the high power side. The accuracy of the simulated pdf distribution enables the verification of analytic procedures for calculating the distribution. The observed discrepancy between the simulated and analytic results suggests that the second-order contributions are important and should be more precisely determined in future studies.

\section{ACKNOWLEDGMENT}

B. Tromborg thanks F. Öhman and A. Bilenca for helpful discussions and the University of Waterloo for a visiting professorship for three months in 2007.

\section{REFERENCES}

[1] B. A. Berg and T. Neuhaus, "Multicanonical ensemble: A new approach to simulate first-order phase transition," Phys. Rev. Lett., vol. 68, pp. 9-12, 1992.

[2] B. A. Berg, "Introduction to multicanonical Monte Carlo simulations," Fields Inst. Commun., vol. 26, pp. 1-24, 2000.

[3] M. Reimer, A. Awadalla, D. Yevick, and T. Lu, "Alignment methods for biased multicanonical sampling," J. Opt. Soc. Am. A, vol. 24, no. 8, pp. 2474-2479, 2007.

[4] D. Yevick, "Multicanonical communication system modeling-Application to PMD statistics," IEEE Photon. Technol. Lett., vol. 14, pp. 1512-1514, Nov. 2002.

[5] D. Yevick, "The accuracy of multicanonical system models," IEEE Photon. Technol. Lett., vol. 15, pp. 224-226, Feb. 2003.

[6] T. Lu and D. Yevick, "Biased multicanonical sampling," IEEE Photon. Technol. Lett., vol. 17, pp. 1420-1422, Dec. 2005.

[7] A. O. Lima, I. T. Lima, and C. R. Menyuk, "Error estimation in multicanonical Monte Carlo simulations with applications to polarizationmode-dispersion emulators," J. Lightw. Technol., vol. 23, no. 11, pp. 3781-3789, Nov. 2005

[8] D. Yevick and T. Lu, "Improved multicanonical algorithms," J. Opt. Soc. Am. A, vol. 23, pp. 2912-2918, 2006.

[9] C. R. Menyuk, "Statistical errors in biasing Monte Carlo simulations with applications to polarization-mode dispersion compensators," IEEE J. Lightw. Technol., vol. 24, no. 11, pp. 4184-4196, Nov. 2006.

[10] R. Holzlöhner and C. R. Menyuk, "Use of multicanonical Monte Carlo simulations to obtain accurate bit error rates in optical communication systems," Opt. Lett., vol. 28, pp. 1894-1896, 2003.

[11] W. Pellegrini, J. Zweck, C. R. Menyuk, and R. Holzlöhner, "Computation of bit error ratios for a dense WDM system using the noise covariance matrix and multicanonical Monte Carlo methods," IEEE Photon. Technol. Lett., vol. 17, pp. 1644-1646, Aug. 2005.

[12] A. Bilenca and G. Eisenstein, "Statistical noise properties of an optical pulse propagating in a nonlinear semiconductor optical amplifier," IEEE J. Quantum Electron., vol. 41, pp. 36-44, Jan. 2005.

[13] A. Bilenca and G. Eisenstein, "Fokker-Planck and Langevin analyses of noise accompanying the amplification of optical pulses in semiconductor optical amplifiers," J. Opt. Soc. Am. B, vol. 22, no. 8, pp. 1632-1639, 2005.

[14] T. I. Lakoba, "Multicanonical Monte Carlo study of the BER of an all-optically 2R regenerated signal," IEEE J. Sel. Topics Quantum Electron., vol. 14, no. 3, pp. 599-609, May/Jun. 2008.
[15] T. I. Lakoba and M. Vasilyev, "A comparative study of noisy signal evolution in $2 \mathrm{R}$ all-optical regenerators with normal and anomalous average dispersions using an accelerated multicanonical Monte Carlo method," Opt. Exp., vol. 16, no. 22, pp. 17714-17728, 2008.

[16] T. I. Lakoba, "BER degradation by signal-reshaping processors with noninstantaneous response," IEEE J. Lightw. Technol., vol. 27, no. 10, pp. 1379-1386, Jul. 2009.

[17] F.Öhman, J. Mørk, and B. Tromborg, "Output power PDF of a saturated semiconductor optical amplifier: Second order noise contributions by path integral method," IEEE J. Quantum Electron., vol. 43, pp. 1188-1197, Dec. 2007

[18] G. P. Agrawal and N. A. Olsson, "Self-phase modulation and spectral broadening of optical pulses in semiconductor laser amplifiers," IEEE J. Quantum Electron., vol. 25, pp. 2297-2306, Nov. 1989.

[19] M. Shtaif, B. Tromborg, and G. Eisenstein, "Noise spectra of semiconductor optical amplifiers: Relation between semiclassical and quantum descriptions," IEEE J. Quantum Electron., vol. 34, pp. 869-878, May 1998.

[20] F.Öhman, S. Bischoff, B. Tromborg, and J. Mørk, "Noise and regeneration in semiconductor waveguides with saturable gain and absorption,” IEEE J. Quantum Electron., vol. 40, pp. 245-255, Mar. 2004.

[21] D. Cassioli, S. Scotti, and A. Mecozzi, "A time-domain computer simulator of the nonlinear response of semiconductor optical amplifiers," IEEE J. Quantum Electron., vol. 36, pp. 1072-1080, Sep. 2000.

[22] N. Metropolis, A. W. Rosenbluth, A. H. Teller, and E. Teller, "Equations of state calculations by fast computing machines," J. Chem. Phys., vol. 21, no. 6, pp. 1087-1092, 1953.

[23] W. K. Hastings, "Monte Carlo sampling methods using Markov chains and their applications," Biometrica, vol. 57, no. 1, pp. 97-109, 1970.

[24] B. Berg, "Algorithmic aspects of multicanonical simulations," Nucl. Phys. B. Proc. Suppl., vol. 63, pp. 982-984, 1998.

Bjarne Tromborg received the M.Sc. degree in physics and mathematics from the Niels Bohr Institute, Copenhagen, Denmark, in 1968, and continued as university researcher in high-energy particle physics.

In 1979, he joined the research laboratory of the Danish Teleadministrations. He was the Head of Optical Communications Department at Tele Danmark Research from 1987 to 1995, an Adjunct Professor at the Niels Bohr Institute from 1991 to 2001, a Project Manager at Tele Danmark R\&D between 1996 and 1998, and took a leave of absence at Technion, Haifa, Israel, in 1997. He was with COM, Technical University of Denmark, from 1999 until he retired in June 2006, most of the time as Research Professor in charge of coordination of modeling of components and systems for optical communications. He has coauthored a research monograph and more than 100 journal and conference papers, mostly on physics and technology of optoelectronic devices.

Prof. Tromborg was awarded the Electro-prize from the Danish Society of Engineers, in 1981, and the Senior Prize 2005 from the Danish Optical Society. He was an Associate Editor of IEEE JOURNAL OF QUANTUM ELECTRONICS during 2003-2006.

Michael Reimer (S'07) received the B.Sc. degree (with honors) in physics (co-op) from the University of British Columbia, Vancouver, BC, Canada, in 1999, and the M.Sc. degree in applied physics from the University of Waterloo, Waterloo, ON, Canada, in 2007, where he is currently working toward the Ph.D. degree in applied physics under the supervision of Prof. D. Yevick.

Prior to 2005, he was with the next generation optical network design team at Nortel Networks.

Mr. Reimer has received the Outstanding Achievement in Graduate Studies distinction from the University of Waterloo for top academic standing at the graduate level in the Faculty of Science and holds the Alexander Graham Bell Canada Graduate Scholarship (Doctoral) from the Natural Sciences and Engineering Research Council of Canada (NSERC).

David Yevick (SM'86-F'96) received the Ph.D. degree from Princeton University, Princeton, NJ, in 1977.

He has been a Professor of physics at the University of Waterloo, Waterloo, ON, Canada, since 1999. He has authored or coauthored more than 165 papers on the theory and numerical analysis of optical fibers and integrated optic and semiconductor laser components as well as on computational and experimental procedures for optical communication system characterization.

Dr. Yevick is a Fellow of the American Physical Society and the Optical Society of America. 